\title{
Embolia balística venosa retrógrada transtorácica: relato de caso e revisão da literatura
}

\author{
Transthoracic retrograde venous bullet embolism: case report and \\ review of the literature
}

\begin{abstract}
Alexandre de Tarso Machado ${ }^{1}$, Ricardo Jayme Procópio ${ }^{1}$, Francesco Botelho Evangelista ${ }^{1}$, Gustavo Henrique Dumont Kleinsorge ${ }^{2}$, Cristina Toledo Afonso ${ }^{3}$, Túlio Pinho Navarro ${ }^{4}$
\end{abstract}

\section{Resumo}

Êmbolo balístico é um fenômeno de ocorrência rara no atendimento ao traumatizado. Geralmente, são pouco sintomáticos ou assintomáticos, e o tratamento desses pacientes, apesar de estar em constante evolução, é ainda controverso. A abordagem endovascular tem se destacado como modalidade de tratamento para esse tipo de embolia com baixa morbimortalidade. Este artigo relata o caso de um paciente de 30 anos, do sexo masculino, vítima de ferimento por arma de fogo com múltiplos orifícios de entrada em tórax, submetido com sucesso à retirada do projétil por acesso endovascular após tentativa frustrada de retirada por toracotomia.

Palavras-chave: Tratamento, embolia, projétil.

\section{Introdução}

O primeiro caso de embolia vascular por corpo estranho foi descrido por Davis em 1834 em criança com fragmento de madeira embolizado para coração ${ }^{1}$. Desde então, foram relatados na literatura diversos outros tipos de materiais relacionados à embolia pós-traumática ou iatrogênica, como agulhas, ponta de cateter, fragmentos metálicos e projéteis de arma de fogo ${ }^{1-12}$.

A embolia balística é fenômeno raro nos traumas por arma de fogo. Sua ocorrência está relacionada aos períodos de guerra e violência social ${ }^{2-4}$.

Na maioria das vezes, os pacientes são assintomáticos. As manifestações clínicas, quando presentes, sofrem

\begin{abstract}
Bullet embolism is a rare event when providing care to traumatized patients. These cases usually present with few symptoms or are asymptomatic, and treatment is controversial, in spite of the evolution observed. The endovascular approach has stood out as a treatment modality for this type of embolism with low morbidity and mortality rates. This article reports the case of a 30 -year-old male patient victim of gunshot thorax injury with multiple entrance signs who was successfully submitted to bullet removal by endovascular technique after failed attempt by thoracotomy.
\end{abstract}

Keywords: Treatment, embolism, bullet.

influência de fatores como localização do projétil e presença de complicações trombóticas, isquêmicas ou hemorrágicas. A localização do projétil por exames de imagem no pré e no intra-operatório é importante para definição diagnóstica e terapêutica. O tratamento desses pacientes, apesar de estar em constante evolução, é ainda controverso. A abordagem endovascular tem se destacado como modalidade de tratamento para esse tipo de embolia.

\section{Descrição do caso}

Homem, 30 anos, vítima de trauma por arma de fogo, com múltiplos disparos na região do tórax.

Admitido com vias aéreas pérvias, taquidispnéico, sons respiratórios diminuídos bilateralmente, choque

1. Médico, Serviço de Cirurgia Vascular e Endovascular, Hospital das Clínicas, Universidade Federal de Minas Gerais, (UFMG), Belo Horizonte, MG.

2. Residente, Cirurgia Vascular, Hospital das Clínicas, UFMG, Belo Horizonte, MG.

3. Residente, Cirurgia Geral, Hospital das Clínicas, UFMG, Belo Horizonte, MG.

4. Doutor. Coordenador, Serviço de Cirurgia Vascular e Endovascular, Hospital das Clínicas, UFMG, Belo Horizonte, MG.

Não foram declarados conflitos de interesse associados à publicação deste artigo.

Artigo submetido em 27.08.08, aceito em 14.10.08.

J Vasc Bras. 2008;7(4):393-396.

Copyright $\odot 2008$ by Sociedade Brasileira de Angiologia e de Cirurgia Vascular 


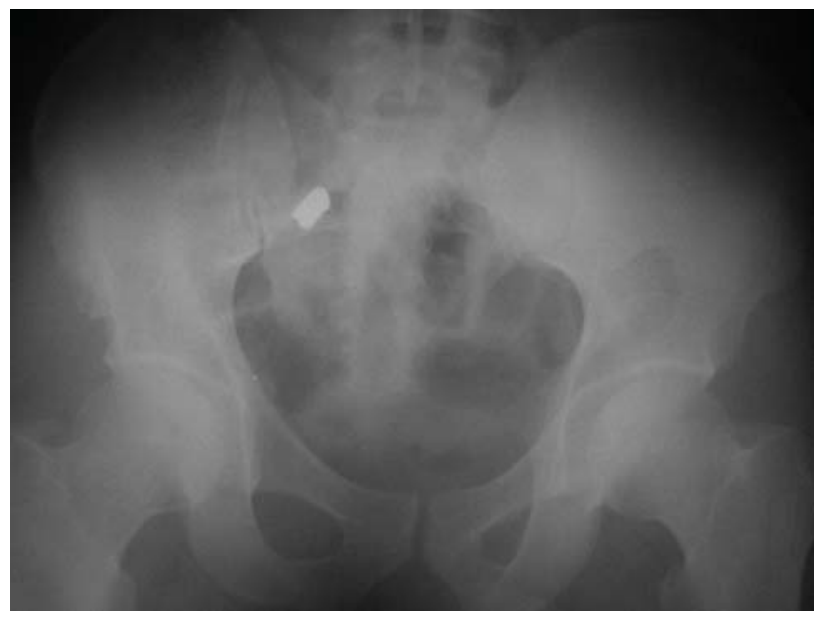

Figura 1 - Radiografia simples de pelve identificando projétil em região sacral direita

hipovolêmico e rebaixamento do nível da consciência (Glasgow 9/15). Realizou-se reposição volêmica com soro fisiológico e drenagem de tórax bilateralmente.

Apresentava orifícios de entrada nas regiões paravertebrais torácicas, bilateralmente, orifício de saída em linha axilar posterior esquerda e lesão transfixante em membro superior esquerdo. Em tomografia de tórax, foi evidenciado projétil em ápice cardíaco, sendo indicado tratamento cirúrgico. Foi submetido a esternotomia, em que não foi constatada lesão externa em coração, sendo o projétil palpado dentro do ventrículo direito. No intraoperatório, ocorreu deslocamento acidental do projétil, inviabilizando sua retirada. Em radiografia simples de tórax intra-operatória, o projétil foi identificado em topografia da veia cava retro-hepática, o que motivou a interrupção da cirurgia.

Posteriormente, em nova radiografia de abdome, localizou-se o projétil na pelve (Figura 1). Optou-se, então, por tratamento endovascular, que foi realizado 10 dias após o trauma.

$\mathrm{Na}$ intervenção, através do acesso venoso da veia femoral comum direita, flebografia pélvica identificou o projétil alojado na veia ilíaca interna direita (Figura 2).

Realizou-se a captura do mesmo com cateter tipo cesta (basket), que foi então tracionado até o local da punção (Figura 3). O acesso cirúrgico foi feito via vasos

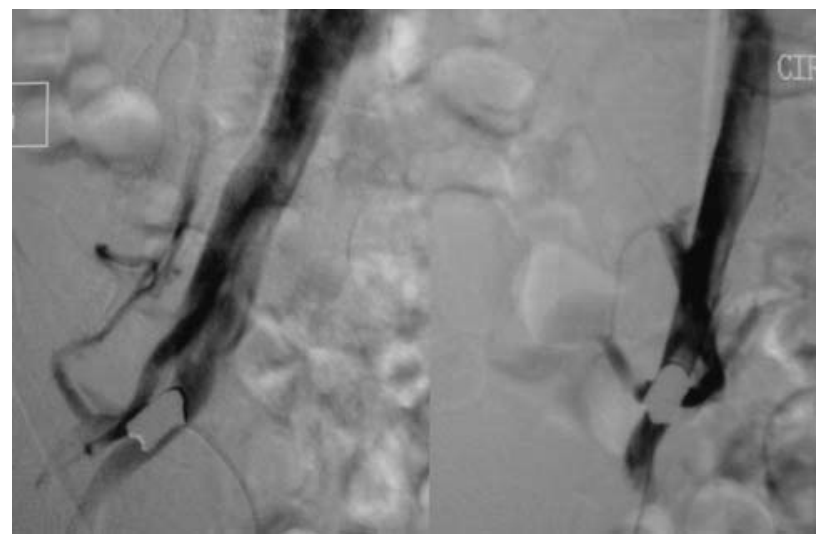

Figura 2 - Flebografia pélvica após cateterismo seletivo de veia ilíaca interna direita

femorais e flebotomia transversa com retirada do projétil, seguida por fleborrafia. Recebeu alta no terceiro dia pós-procedimento.

\section{Discussão}

Embolia balística deve ser considerada nos pacientes que apresentam orifício de entrada de arma de fogo sem orifício de saída correspondente nos casos em que o projétil não é observado radiograficamente na localização esperada ${ }^{4,5}$.

Pacientes são assintomáticos em cerca de $70 \%$ dos $\operatorname{casos}^{1,6}$.Os sintomas, quando presentes, dependem da localização do êmbolo e associação com complicações como dor, dispnéia, astenia, hemorragia, trombose, arritmias, disfunção valvar, endocardite, sepse, erosão ou oclusão vascular ${ }^{3,4,7,8}$

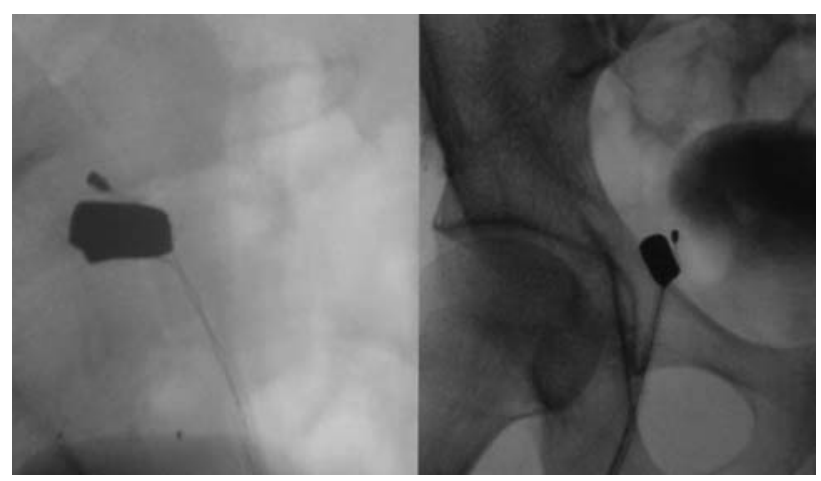

Figura 3 - Captura e remoção do projétil com cesta tipo basket 
A penetração intravascular do projétil pode ser resultado de injúria direta ao coração ou a outros sítios vasculares $^{9}$. Após a penetração, o projétil pode migrar pela via intravascular, que geralmente ocorre imediatamente após o trauma. No entanto, já foi descrita migração após 14 anos do evento. Outros autores descrevem migração durante o ato cirúrgico, como ocorreu no caso relatado. Essa migração depende de fatores como peso, velocidade, tamanho e forma do êmbolo, posição do paciente, ação da gravidade, movimentos musculares e respiratórios, anatomia vascular e velocidade de fluxo do vaso envolvido $^{3,7,10}$.

O deslocamento do projétil pode ser favorecido pela baixa velocidade e pequeno calibre do projétil, envolvendo a circulação pulmonar ou sistêmica, via sistema venoso ou arterial ${ }^{9}$.

O nível de impactação depende do calibre do êmbolo e do diâmetro do vaso e ocorre principalmente nas bifurcações vasculares, por serem os locais de diminuição abrupta de calibre. $\mathrm{O}$ vaso mais comumente ocluído na embolia arterial é a artéria femoral (30 a 50\% dos (asos) $)^{3,11,12}$.

No paciente apresentado, o projétil foi encontrado inicialmente no ventrículo direito, e presume-se que ele tenha entrado no coração por algum ramo da artéria pulmonar e não por lesão direta ao mesmo, pois durante a exploração cirúrgica não foram observadas lesões externas cardíacas. Com a manipulação durante a operação, o projétil migrou, seguiu retrogradamente em direção à veia cava retro-hepática e posteriormente à veia ilíaca comum direita.

Métodos de imagem para a localização do projétil, como radiografia simples, tomografia computadorizada, ecocardiografia ou arteriografia são fundamentais para se confirmar o diagnóstico e se programar a terapêutica ${ }^{3}$. Esses exames são ainda importantes no préoperatório para demonstrar eventual migração do êmbolo durante a manipulação do paciente ${ }^{9}$.

Existem controvérsias na literatura quanto ao tratamento da embolia balística. Entretanto, a maioria dos autores recomenda tratamento conservador em pacientes assintomáticos e indica a remoção cirúrgica do êmbolo quando existem sintomas ou possibilidade de ocorrência de complicações, como embolização para áreas de risco. O paciente em questão não apresentava sintomas diretamente relacionados à embolia, mas havia possibilidade de ocorrência de complicações, como trombose venosa profunda ou embolia pulmonar, o que justificou o tratamento cirúrgico.

A escolha do procedimento cirúrgico baseia-se na exata localização do projétil e nas condições clínicas de cada paciente. Dentre as diversas modalidades de tratamento disponíveis, a cirurgia endovascular tem ganhado cada vez mais espaço por suas vantagens em relação à cirurgia aberta, como o uso de anestesia local e a menor morbimortalidade ${ }^{3,4,7-9}$. A cirurgia endovascular tem sido também considerada como primeira opção de tratamento para êmbolos móveis, sendo a técnica mais freqüentemente utilizada a remoção percutânea por cateteres do tipo cesta (basket) ou laço (snare ${ }^{11}$. O tratamento endovascular com cateter tipo cesta foi empregado com sucesso no caso relatado.

\section{Conclusão}

A embolia balística é evento raro que deve ser considerado nos casos de trauma por arma de fogo em que não há orifício de saída para o projétil e sem identificação do mesmo pela radiografia simples no local esperado. Na maioria das vezes, são assintomáticos e necessitam apenas de tratamento conservador, mas podem ser adequadamente tratados com técnicas minimamente invasivas com baixa morbimortalidade, quando necessário.

\section{Referências}

1. Nagy KK, Massad M, Fildes J, Reyes H. Missile embolization revisited: a rationale for selective management. Am Surg. 1994;60:975-9.

2. Starling SV, Maia E, Drumond DAF. Embolo balístico na artéria pulmonar. Trauma. 2007;10:73-98.

3. Demirkilic U, Yilmaz AT, Tatar H, Ozturk OY. Bullet embolism to the pulmonary artery. Interact Cardiovasc Thorac Surg. 2004;3:356-8.

4. Cysne E, Souza EG, Freitas E, et al. Bullet embolism into the cardiovascular system. Tex Heart Inst J. 1982;9:75-80.

5. Duncan IC, Fourie PA. Embolization of a bullet in the internal carotid artery. AJR Am J Roentgenol. 2002;178:1572-3.

6. Chen JJ, Mirvis SE, Shanmuganathan H. MDCT diagnosis and endovascular management of bullet embolization to the heart. Am Soc Emergency Radiol. 2007;14:127-30. 
7. Padula RT, Sandler SC, Camishion RC. Delayed bullet embolization to the heart following abdominal gunshot wound. Ann Surg. 1969;169:599-602.

8. Symbas PN, Harlaftis N. Bullet emboli in the pulmonary and systemic arteries. Ann Surg. 1977;185:318-20.

9. Actis Dato GM, Arslanian A, Di Marzio P, Filosso PL, Ruffini E. Posttraumatic and iatrogenic foreign bodies in the heart: report of fourteen cases and review of literature. J Thorac Cardiovasc Surg. 2003;126:408-14.

10. Saltzstein EC, Freeark RJ. Bullet embolism to the right axillary artery following gunshot wound of the heart. Ann Surg. 1963;158:65-9.
11. Bertoldo U, Enrichens F, Comba A, Ghiselli G, Vaccarisi S, Ferraris M. Retrograde venous bullet embolism: a rare occurrence-case report and literature review. J Trauma. 2004;57:187-92.

12. Garzon A, Gliedman M. Peripheral embolization of a bullet following perforation of the thoracic aorta. Ann Surg. 1964;160:901-4.

Correspondência:

Alexandre de Tarso Machado

Av. Afonso Pena, 2541

CEP 30130-007 - Belo Horizonte, MG

Tel.: (31) 3271.1829

E-mail: alextm@medicina.ufmg.br

\section{Colega Associado da SBACV}

Você está convidado a participar do crescimento e consolidação do J Vasc Bras - como autor, leitor ou revisor. Leia e divulgue; conheça as normas e submeta seus trabalhos.

Jornal Vascular Brasileiro - Secretarial Editorial Rua Maraguape, 72, loja 01

CEP 90690-380 - Porto Alegre - RS - Fone (51) 3012-0575

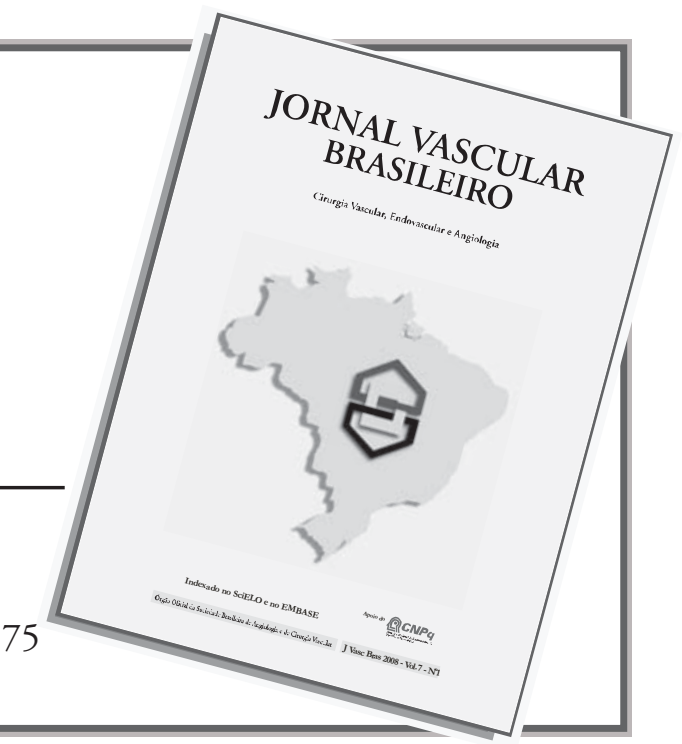

